\title{
Simultaneous Swelling and Stress Relaxation Behavior of Uniaxially Stretched Polymer Gels
}

\author{
Toshikazu Takigawa, Kenji Urayama, Yoshiro Morino, \\ and Toshiro MASUDA
}

Research Center for Biomedical Engineering, Kyoto University, Shogoin, Sakyo-ku, Kyoto 606-01, Japan

(Received December 18, 1992)

\begin{abstract}
We have evaluated the swelling ratio and the stress value in equilibrium $\left(\tilde{\sigma}_{x \infty}\right)$ after uniaxial elongation of polymer gels, employing the Flory-type of the Gibbs free energy formula. Poisson's ratio in equilibrium $\left(\mu_{\infty}\right)$, which determines the degree of swelling under tension, is estimated to be $1 / 6$ so far as the applied strain is not large. The expression for the equilibrium stress $\tilde{\sigma}_{x \infty}$ is derived by the free energy. We have also presented a swelling kinetics theory for the rectangular gels, which describes the change of volume of the gels after uniaxial elongation. The stress relaxation of the gel obeys the same kinetics as the swelling process from the initial to the final values of stress. The experimental values of $\mu_{\infty}$ and $\tilde{\sigma}_{x \infty}$ as equilibrium properties for poly(acrylamide) gels agreed fairly well with the theory. The swelling and stress relaxation kinetics for PAAm gels were also well described by the theory presented in this work.

KEY WORDS Degree of Swelling / Stress Relaxation / Swelling Kinetics /

Poisson's Ratio / Poly(acrylamide) Gel /
\end{abstract}

As well known, polymer gel are unique soft materials and are being applied to biomedical uses. ${ }^{1-3}$ Besides the studies for applications, the basic aspects of the physical properties of the gels, especially volume phase transition ${ }^{4-9}$ where the gel volume changes discontinuously in a narrow range of temperature, $\mathrm{pH}$ and so on, have been extensively studied. The equilibrium swelling behavior of gels has also been investigated by many researchers. ${ }^{10-15}$ However, the studies on swelling under tension are quite a few at present. ${ }^{16,17}$ Concerning the mechanical properties of gels, on the other hand, there are many studies. ${ }^{18-23}$ For example, stress-strain behavior has been examined for various kinds of gels. ${ }^{3,20,24,25}$ Since the studies are limited to the properties at short times, however, the mechanical properties of gels at long times are still unclear because of only a few experimental and theoretical studies. ${ }^{23,26}$ When a gel is stretched, the free energy of the gel system will change to attain a new equilibrium state under tension. This may cause a'volume change of the gel. Tanaka et al. have reported ${ }^{27,28}$ that the relaxation time for swelling is determined by the diffusion constant and sample size of freely swelling gel systems. The same concept may be applicable to the swelling of the stretched gel systems, that is, the swelling of the stretched gel also takes long time until reaching the equilibrium size. As can be easily imagined, the swelling behavior strongly affects mechanical properties of polymer gels. We have already reported $^{29}$ the mechanical properties at short times, such as the stress-strain behavior and Poisson's ratio $(\mu)$ of poly(vinyl alcohol) (PVA) gels. The mechanical properties of the gels could be regarded as that of a swollen rubber; $\mu$ was very close to 0.5 independently of the strain rates. This fact implies that $\mu$ of the gels is a "material constant," and happened because 
the reciprocal of the experimental strain rate, which can be considered as a time-scale of the experiment, is much shorter than the time required for the swelling and volume change. It is very important to investigate effects of the degree of swelling on the mechanical properties of gels under tension for further understanding the properties of gels. In this study, we investigate the equilibrium swelling behavior of gels under tension, and also the dymanics of swelling and stress relaxation at long times after uniaxial elongation. This paper is divided into four sections. In the section of THEORY, we theoretically discuss the thermodynamics and kinetics of uniaxially stretched gels. The experimental details for the preparation of poly(acrylamide) (PAAm) gels and the measurements are described in EXPERIMENTAL section. The theoretical calculations, experimental results, and the comparison between theory and experiment are shown in RESULTS AND DISCUSSION.

\section{THEORY}

\section{Swelling Behavior of the Uniaxially Stretched Gels}

We consider here uniaxial elongation process of isotropic gels from a freely swollen state, i.e., the state without external tension. The state is referred to as the reference state. Using Flory-type expression, ${ }^{10}$ the Gibbs free energy $(F)$ of the uniaxially stretched gel in $x$-direction can be expressed as ${ }^{7}$

$$
\begin{aligned}
F & =F_{0}+N_{\mathrm{s}} k_{\mathrm{B}} T[\ln (1-\phi)+x \phi] \\
& +\frac{N_{\mathrm{c}} k_{\mathrm{B}} T}{2}\left[\alpha_{x}^{2}+\alpha_{y}^{2}+\alpha_{z}^{2}-3-\ln \frac{\alpha_{x} \alpha_{y} \alpha_{z}}{V_{0}}\right] \\
& -f_{x} l_{x 0}\left(\alpha_{x}-1\right)
\end{aligned}
$$

where $F_{0}$ is the free energy of pure polymer and solvent, $N_{\mathrm{c}}$ and $N_{\mathrm{s}}$ respectively the number of active chains and of solvent moleculues in the reference state, $V_{0}$ the volume in the reference state, $\phi$ the polymer volume fraction,
$k_{\mathrm{B}}$ the Boltzmann constant, $T$ the absolute temperature, $f_{x}$ the external force, $l_{x 0}$ the initial length of the gel in $x$-direction, and $\chi$ the polymer-solvent interaction parameter. The quantity. $\alpha_{i}(i=x, y$, and $z)$ is defined by

$$
\alpha_{i}=l_{i} / l_{i 0}
$$

where $l_{i}$ is the sample dimension in the $i$-direction in the stretched state, $l_{i 0}$ that in the reference state, and $\alpha_{x} \alpha_{y} \alpha_{z}=V / V_{0}=\phi_{0} / \phi$, where $V$ is the volume in the deformed state and $\phi_{0}$ is the value of $\phi$ in the reference state. The shear modulus $G_{0}$ in the reference state is written by $G_{0}=N_{\mathrm{c}} k_{\mathrm{B}} T$. Without losing generality, we can set $l_{i 0}=1$ and accordingly $V_{0}=1$. In this case, the quantity $f_{x}$ can be considered as the nominal stress acting in $x$-direction. Regarding $F$ as a function of $\alpha_{i}, \Pi_{i}(i, j, k=x$, $y$, and $z$ ) can be defined as

$$
\Pi_{i}=-\frac{1}{\alpha_{j} \alpha_{k}}\left(\frac{\partial F}{\partial \alpha_{i}}\right)
$$

$\Pi_{i}$ is the swelling stress acting normally on the surface of the gel perpendicular to $i$-axis, and $\Pi_{i}=0$ in equilibrium, namely,

$$
\begin{aligned}
-\Pi_{x}= & \frac{k_{\mathrm{B}} T}{v_{\mathrm{s}}}\left[\ln (1-\phi)+\phi+x \phi^{2}\right] \\
& +\frac{N_{\mathrm{c}} k_{\mathrm{B}} T}{2 \alpha_{y} \alpha_{z}}\left(2 \alpha_{x}-\frac{1}{\alpha_{x}}\right)-\frac{f_{x}}{\alpha_{y} \alpha_{z}}=0 \\
-\Pi_{y}= & \frac{k_{\mathrm{B}} T}{v_{\mathrm{s}}}\left[\ln (1-\phi)+\phi+x \phi^{2}\right] \\
& +\frac{N_{\mathrm{c}} k_{\mathrm{B}} T}{2 \alpha_{z} \alpha_{x}}\left(2 \alpha_{y}-\frac{1}{\alpha_{y}}\right)=0 \\
-\Pi_{z}= & \frac{k_{\mathrm{B}} T}{v_{\mathrm{s}}}\left[\ln (1-\phi)+\phi+x \phi^{2}\right] \\
& +\frac{N_{\mathrm{c}} k_{\mathrm{B}} T}{2 \alpha_{x} \alpha_{y}}\left(2 \alpha_{z}-\frac{1}{\alpha_{z}}\right)=0
\end{aligned}
$$

where $v_{\mathrm{s}}$ is the volume of a solvent molecule and is given by $v_{\mathrm{s}}=(1-\phi) V / N_{\mathrm{s}}$. The average swelling pressure $(\Pi)$ is written by $\Pi=(1 / 3) \Sigma \Pi_{i}$, and $\Pi=0$ is also satisfied in 
equilibrium. The quantity $\tilde{\sigma}_{x \infty}$, the external stress exerted on the gel in $x$-direction in equilibrium, is expressed by

$$
\begin{aligned}
\tilde{\sigma}_{x \infty}=\frac{f_{x}}{\alpha_{y} \alpha_{z}}= & \frac{k_{\mathrm{B}} T}{v_{\mathrm{s}}}\left[\ln (1-\phi)+\phi+\chi \phi^{2}\right] \\
& +\frac{N_{\mathrm{c}} k_{\mathrm{B}} T}{2 \alpha_{y} \alpha_{z}}\left(2 \alpha_{x}-\frac{1}{\alpha_{x}}\right)
\end{aligned}
$$

In the reference state, $\tilde{\sigma}_{x \infty}=0$ and also $\Pi_{i}=0$. Then, we obtain

$$
N_{\mathrm{c}}=-\frac{2}{v_{\mathrm{s}}}\left[\ln \left(1-\phi_{0}\right)+\phi_{0}+x \phi_{0}^{2}\right]
$$

Hereafter, we deal with the gels with $\phi_{0} \ll 1$, and also $\phi \ll 1$. When $k \ll 1, \ln (1-k)$ can be expanded as $-k-(1 / 2) k^{2}+\mathrm{O}\left(k^{3}\right)$. Using the approximation, we try to obtain the expressions for $\mu, \Pi_{i}$, and $\tilde{\sigma}_{x \infty}-\Pi_{x}$ in equilibrium. Since we consider the uniaxial elongation of isotropic gels in $x$-direction, $\alpha_{y}=\alpha_{z}$. Then, eq 5 coincides with eq 6 , and is expressed by

$$
\begin{aligned}
-\Pi_{y}= & \frac{k_{\mathrm{B}} T}{v_{\mathrm{s}}}\left(x-\frac{1}{2}\right)\left(\frac{\phi_{0}}{V}\right)^{2} \\
& +\frac{N_{\mathrm{c}} k_{\mathrm{B}} T}{2}\left(\frac{2}{\alpha_{x}}-\frac{1}{V}\right)=0
\end{aligned}
$$

Equation 8 is also written by

$$
N_{\mathrm{c}}=\frac{2 \phi_{0}^{2}}{v_{\mathrm{s}}}\left(\frac{1}{2}-\chi\right)
$$

Combining eq 9 with eq 10 , we have

$$
\frac{1}{V^{2}}+\frac{1}{V}-\frac{2}{\alpha_{x}}=0
$$

From this equation, $\alpha_{y}^{2}$ and $\alpha_{z}^{2}$ are given by

$$
\alpha_{y}^{2}=\alpha_{z}^{2}=\left(\alpha_{x}+\sqrt{\alpha_{x}^{2}+8 \alpha_{x}}\right) / 4 \alpha_{x}
$$

Since the strain in $i$-direction $\left(\varepsilon_{i}\right)$ for the gel is defined by

$$
\varepsilon_{i}=\alpha_{i}-1
$$

we can linearize eq 12 using eq 13 when the strain is small. At equilibrium $\varepsilon_{y}$ and $\varepsilon_{z}$ are written by

$$
\varepsilon_{y}=\varepsilon_{z}=-\frac{1}{6} \varepsilon_{x}
$$

Equation 14 shows that the value of Poisson's ratio $\mu$ at equilibrium $\left(\mu_{\infty}\right)$ is $1 / 6$. Using the value of $\mu_{\infty}$ and the relation $K_{\mathrm{os}}=2\left(1+\mu_{\infty}\right) G_{0} /$ $3\left(1-2 \mu_{\infty}\right)$, the osmotic bulk modulus $K_{\mathrm{os}}$ is given by $K_{\mathrm{os}}=(7 / 6) G_{0}$. The linearized expressions for $-\Pi_{i}(i=y, z)$ and $\tilde{\sigma}_{x \infty}-\Pi_{x}$ at fixed $\varepsilon_{x}$ are given by

$$
\begin{aligned}
& -\Pi_{y}=\frac{1}{2} G_{0} \varepsilon_{x}+\frac{3}{2} G_{0}\left(\varepsilon_{y}+\varepsilon_{z}\right) \\
& -\Pi_{z}=\frac{1}{2} G_{0} \varepsilon_{x}+\frac{3}{2} G_{0}\left(\varepsilon_{y}+\varepsilon_{z}\right) \\
& \tilde{\sigma}_{x \infty}-\Pi_{x}=\frac{5}{2} G_{0} \varepsilon_{x}+\frac{1}{2} G_{0}\left(\varepsilon_{y}+\varepsilon_{z}\right)
\end{aligned}
$$

In the equilibrium state under tension where $\Pi_{x}=0, \tilde{\sigma}_{x \infty}$ is given by

$$
\tilde{\sigma}_{x \infty}=\frac{7}{3} G_{0} \varepsilon_{x}
$$

\section{Swelling Kinetics of Gels after Extension}

After a tension in $x$-direction is applied to a gel, the gel system moves toward a new equilibrium state under tension by expanding in $y$ - and $z$-direction. This accompanies a volume change of the gel. In order to describe the dynamics of the gel swelling, we set the size of the gel in $y$-and $z$-direction in the reference state (i.e., the state without tension) is $a_{\mathrm{r}}$. The small volume element of the gel can be specified by the position vector, $\boldsymbol{r}=(x, y, z)\left(0 \leq y, z \leq a_{\mathrm{r}}\right)$ in the coordinate of the reference state. According to Tanaka et al., ${ }^{27,28}$ the equation, which governs the time $(t)$ dependent motion of the small volume element $(\mathrm{d} x \mathrm{~d} y \mathrm{~d} z)$ at the position of $(x, y, z)$ after instanteneous elongation with $\varepsilon_{x}$ at $t=0$, can be written by

$$
\zeta \frac{\partial \boldsymbol{u}}{\partial t}=\left(K_{\mathrm{os}}+\frac{1}{3} G_{0}\right) \operatorname{grad}(\operatorname{div} \boldsymbol{u})
$$




$$
+G_{0} \nabla^{2} \boldsymbol{u}
$$

Here, $\boldsymbol{u}(x, y, z, t)$ is the displacement vector specified in the reference frame, and $\zeta$ the friction coefficient between gel network and solvent molecules.

Since eq 19 has a complicated form, we simplify the equation. The displacement vector (u) can be generally written by using scalar potential $\left(\Phi / 2 G_{0}\right)$ and vector potential $\left(\boldsymbol{\Psi} / 2 G_{0}\right)$ with $\operatorname{div}\left(\boldsymbol{\Psi} / 2 G_{0}\right)=0$ as

$$
\boldsymbol{u}=\operatorname{grad}\left(\Phi / 2 G_{0}\right)+\operatorname{rot}\left(\boldsymbol{\Psi} / 2 G_{0}\right)
$$

From eq 19 and 20, we obtain

$$
\begin{aligned}
& \frac{\partial}{\partial t}\left(\nabla^{2} \Phi\right)=D_{\mathrm{L}} \nabla^{2}\left(\nabla^{2} \Phi\right) \\
& \frac{\partial}{\partial t}\left(\nabla^{2} \Psi\right)=D_{\mathrm{T}} \nabla^{2}\left(\nabla^{2} \Psi\right)
\end{aligned}
$$

Here, $D_{\mathrm{L}}$ and $D_{\mathrm{T}}$ are the diffusion constants given with $K_{\text {os }}=(7 / 6) G_{0}$ by

$$
\begin{aligned}
& D_{\mathrm{L}}=\left(K_{\mathrm{os}}+\frac{4}{3} G_{0}\right) / \xi=5 G_{0} / 2 \xi \\
& D_{\mathrm{T}}=G_{0} / \xi
\end{aligned}
$$

The diffusion constant, $D_{\mathrm{L}}$, is identical to the collective diffusion constant introduced by Tanaka et al. ${ }^{27,28}$ The quantities, $\nabla^{2} \Phi$ and $\nabla^{2} \boldsymbol{\Psi}$ are given by

$$
\begin{aligned}
& \nabla^{2} \Phi=2 G_{0} \operatorname{div} \boldsymbol{u}=2 G_{0} \operatorname{tr}\left(u_{i j}\right) \\
& \nabla^{2} \boldsymbol{\Psi}=-2 G_{0} \operatorname{rot} \boldsymbol{u}=-4 G_{0} \boldsymbol{\omega}
\end{aligned}
$$

where $\omega$ is the rotation vector, and $u_{i j}$ is the $i j$ component of strain tensor for the small volume element and is defined by $i$ and $j$ components of the displacement vector $\left(u_{i}\right.$ and $\left.u_{j}\right)$ as $u_{i j}=(1 / 2)\left(\partial u_{j} / \partial i+\partial u_{i} / \partial j\right) \cdot{ }^{30}$ Equation 21 corresponds to the longitudinal mode of the diffusion, and eq 22 to the transverse mode. It should be noted that the diffusion of the gels is generally separated into two modes, and each mode can be described by the diffusion equation. The longitudinal mode of the diffusion accompanies the volume change, while the transverse mode does not accompany the volume change but determines the change of shape. We can see from eq 23 and 24 that the diffusion constant for the longitudinal mode is 2.5 times larger than that of the transverse mode.

We deal hereafter only the longitudinal mode of the diffusion, since the quantity of interest is the degree of swelling of gels determined by the longitudinal mode of the diffusion. The swelling after elongation can be assumed to occur in $y$ - and $z$-direction, so that $\boldsymbol{u}(x, y, z, t)$ is independent of $x$. This means that $u_{x y}=u_{y x}=u_{x z}=u_{z x}=0$, and $u_{x x}$ is constant. We rewrite eq 21 by using $\nabla^{2} \Phi=2 G_{0} \operatorname{tr}\left(u_{i j}\right)$ in eq 25 as

$$
\frac{\partial}{\partial t} \operatorname{tr}\left(u_{i j}\right)=D_{\mathbf{L}} \nabla^{2} \operatorname{tr}\left(u_{i j}\right)
$$

It should be noted that this diffusion equation is defined in 2-dimensional space. The volume change (the change of the cross-sectional area in 2-dimensional space) of the gels is determined by the above equation with the initial and boundary conditions given by

$$
\begin{array}{lll}
\operatorname{tr}\left(u_{i j}\right)=\left(\varepsilon_{y 0}+\varepsilon_{z 0}\right) & \text { at } & t=0 \\
\operatorname{tr}\left(u_{i j}\right)=-2 \mu_{\infty} \varepsilon_{x} & \text { at } & \text { boundaries }
\end{array}
$$

The quantities $\varepsilon_{y 0}$ and $\varepsilon_{z 0}$ are the initial values of strain imposed in $y$ and $z$ directions, and are respectively written by using a macroscopic strain in $x$ direction $\left(\varepsilon_{x}\right)$ and $\mu$ at short times $\left(\mu_{0}\right)$ by $\varepsilon_{y 0}=-\mu_{0} \varepsilon_{x}$ and $\varepsilon_{z 0}=-\mu_{0} \varepsilon_{x}$, where $\varepsilon_{x}=u_{x x}$. The value of $\mu_{0}$ is here $1 / 2$. Equation 28 means that the cross-section of the gel shrinks at $t=0$, and eq 29 shows that the osmotic pressure acting on the small element at the boundaries relaxes at any $t(>0)$.

The solution of eq 27 satisfying the above conditions is

$$
\operatorname{tr}\left(u_{i j}\right)=\sum_{m, n}^{\text {odd }} A_{m n} \sin \frac{m \pi y}{a_{\mathrm{r}}} \sin \frac{n \pi z}{a_{\mathrm{r}}} \mathrm{e}^{-k_{m n} t}-2 \mu_{\infty} \varepsilon_{x}
$$


Here, the constants in the equation above are given by

$$
\begin{aligned}
& A_{m n}=\frac{-32\left(\mu_{0}-\mu_{\infty}\right) \varepsilon_{x}}{m n \pi^{2}} \\
& k_{m n}=\frac{D_{\mathrm{L}} \pi^{2}\left(m^{2}+n^{2}\right)}{a_{\mathrm{r}}^{2}}=\frac{m^{2}+n^{2}}{2 \tau_{1}}
\end{aligned}
$$

and $\tau_{1}$ is the longest relaxation time, and $m$ and $n$ are odd integers. The $t$ dependence of the average width of the gel $(a(t))$ in the $y$ - and $z$-direction is determined by

$$
a(t)^{2}=a_{\mathrm{r}}^{2}\left(1+\varepsilon_{y}(t)+\varepsilon_{z}(t)\right)=\int \mathrm{d} S^{\prime}
$$

where $\mathrm{d} S^{\prime}=\mathrm{d} S\left(1+u_{y y}+u_{z z}\right)$ and $\mathrm{d} S=\mathrm{d} y \mathrm{~d} z$. The $t$ dependence of $\varepsilon_{y}$ (or $\varepsilon_{z}$ ) can be written by

$$
\begin{aligned}
& \varepsilon_{y}(t)=\varepsilon_{z}(t)=\frac{1}{2}\left(\frac{a(t)^{2}}{a_{\mathrm{r}}^{2}}-1\right) \\
& =\frac{1}{2 a_{\mathrm{r}}^{2}}\left[\int_{0}^{a_{\mathrm{r}}} \mathrm{d} y \int_{0}^{a_{\mathrm{r}}} \mathrm{d} z\right. \\
& \left.\quad \times\left\{\sum_{m, n}^{\text {odd }} A_{m n} \sin \frac{m \pi y}{a_{\mathrm{r}}} \sin \frac{n \pi z}{a_{\mathrm{r}}} \mathrm{e}^{-k_{m n} t}-2 \mu_{\infty} \varepsilon_{x}\right\}\right]
\end{aligned}
$$

\section{Stress Relaxation of Gels}

We consider the external stress for small volume element with $\mu_{0}=1 / 2\left(\tilde{s}_{x}(x, y, z, t)\right)$. When $\tilde{s}_{x}(x, y, z, t)$ is instanteneously released, the small volume element will shrink in $x$ direction and expand in $y$ - and $z$-direction. We assume that the recovered size in the three directions is identical, and is determined in order to keep the volume constant before and after the stress release. This means that $\tilde{s}_{x}$ is proportional to the difference in $u_{x x}$ between the stretched state and the isotropically swollen state. Using an average strain magnitude, $(1 / 3)\left(u_{x x}+u_{y y}+u_{z z}\right), \tilde{s}_{x}$ can be written by

$$
\begin{aligned}
\tilde{s}_{x} & =3 G_{0}\left(u_{x x}-\frac{u_{x x}+u_{y y}+u_{z z}}{3}\right) \\
& =G_{0}\left(2 u_{x x}-u_{y y}-u_{z z}\right)
\end{aligned}
$$

It should be noted that the average strain magnitude characterizes the new reference state, and is measured from the original reference state, and $u_{x x}$ is a constant $\left(=\varepsilon_{x}\right)$. Finally, the $t$ dependent macroscopic stress, $\tilde{\sigma}_{x}(t)$, can be expressed by

$$
\begin{aligned}
\tilde{\sigma}_{x}(t)=\frac{\int \mathrm{d} S^{\prime} \tilde{s}_{x}(t)}{\int \mathrm{d} S^{\prime}} \cong \frac{\int \mathrm{d} S \tilde{s}_{x}(t)}{\int \mathrm{d} S} \\
=\frac{G_{0}}{a_{\mathrm{r}}^{2}} \int_{0}^{a_{\mathrm{r}}} \mathrm{d} y \int_{0}^{a_{\mathrm{r}}} \mathrm{d} z\left[2\left(1+\mu_{\infty}\right) \varepsilon_{x}\right. \\
\left.-\sum_{m_{z} n}^{\text {odd }} A_{m n} \sin \frac{m \pi y}{a_{\mathrm{r}}} \sin \frac{n \pi z}{a_{\mathrm{r}}} \mathrm{e}^{-k_{m n} t}\right]
\end{aligned}
$$

This agrees with eq 18 at long $t$ limit because $\mu_{\infty}=1 / 6$.

\section{EXPERIMENTAL}

\section{Gel Sample}

Four kinds of PAAm gels were prepared by employing a radical copolymerization technique. The preparation details are as follows: $35.5 \mathrm{~g}$ of acrylamide monomer, $0.154 \mathrm{~g}$ of $N, N^{\prime}$-methylene bis(acrylamide) acting as a crosslinker, and $0.685 \mathrm{~g}$ of ammonium persulfate (initiator) were dissolved in $100 \mathrm{ml}$ of distilled water at room temperature. The solution was casted into $6 \times 6 \times 20 \mathrm{~mm}$ metal mold. Gelation was performed at $33^{\circ} \mathrm{C}$, and gels were kept in the mold for 3 days for curing. Gels were removed from the mold, and transfered in a large amount of water. Gels were maintained at $33^{\circ} \mathrm{C}$ for about 10 days to achieve the equilibrium swelling. The water was exchanged everyday. The four kinds of PAAm gels were prepared in the same way as described above, but they were a litte different from each other in the initial Young's modulus $\left(E_{0}\right)$. The width of the gels before elongation $\left(a_{\mathrm{r}}\right)$ ranged from 9.0 to $9.5 \mathrm{~mm}$. The sample code and $E_{0}$ are summarized in Table I, together with the experimental results. 
Table I. Initial Young's modulus $\left(E_{0}\right)$, the ratio of sample width in the final state to that in the reference state $\left(a_{\infty} / a_{\mathrm{r}}\right)$, the longest relaxation time $\left(\tau_{1}\right)$, and the diffusion constant of the longitudinal mode $\left(D_{\mathrm{L}}\right)$ for poly(acrylamide) (PAAm) gels

\begin{tabular}{cccccc}
\hline & \multicolumn{2}{c}{$E_{0}$} & & & \\
Sample & & $a_{\infty} / a_{\mathrm{r}}$ & $\tau_{1} / 10^{4} \mathrm{~s}$ & $D_{\mathbf{L}}$ \\
\cline { 2 - 5 } & $10^{4} \mathrm{~Pa}$ & & & & $10^{-7} \mathrm{~cm}^{2} \mathrm{~s}^{-1}$ \\
\hline AG1 & 2.3 & 0.973 & $15^{\mathrm{a}}$ & $18^{\mathrm{b}}$ & 2.5 \\
AG2 & 4.1 & 0.979 & $6.0^{\mathrm{a}}$ & $7.5^{\mathrm{b}}$ & 5.5 \\
AG3 & 3.8 & 0.986 & $6.6^{\mathrm{a}}$ & $6.2^{\mathrm{b}}$ & 7.4 \\
AG4 & 3.5 & 0.984 & $9.4^{\mathrm{a}}$ & $9.1^{\mathrm{b}}$ & 4.5 \\
\hline
\end{tabular}

a Measured by swelling experiment.

b Measured by stress relaxation.

\section{Measurements}

Uniaxial elongation of PAAm gels was performed in water by using an Orientec RTM-250 tensile tester with a specially designed bath at $25^{\circ} \mathrm{C}$. The extension process of the samples was recorded with video camera. The value of $\alpha_{x}$ in local level was determined by measuring the distance between the two marked points in the central region of the sample. The gel sample was elongated to globally $\alpha_{x}=1.1$ at cross-head speed of $v=3 \mathrm{mmmin}^{-1}$, and was kept under the elongated state. The time $t=0$ was taken as the time when the elongation was stopped. The width $(a)$ was measured on the video monitor screen as a function of $t$. The width at $t=0$ was designated by $a_{0}$. The $t$ dependence of the width in the one direction (we denote here the direction as $y$ ) was measured after the $x$-directional elongation, and the width in the other direction (z-direction) was assumed to be identical to that in $y$-direction. The applied force $f_{x}$ in $x$-direction was monitored by recorder. The strains $\varepsilon_{x}, \varepsilon_{y}$ were calculated by $\varepsilon_{x}=\alpha_{x}-1$, and $\varepsilon_{y}=a / a_{\mathrm{r}}-1$. The stress $\tilde{\sigma}_{x}$ was calculated by $\tilde{\sigma}_{x}(t)=F(t) / S(t)$ where $F(t)$ is the force and $S(t)$ is the cross-sectional area of the gels at time $t$. Actually $\tilde{\sigma}_{x}$ was almost identical to the engineering stress because the applied strain was small. $S(t)$ was assumed to be $a^{2}$.

\section{RESULTS AND DISCUSSION}

As stated in the experimental section, we elongated the gel uniaxially (in $x$-direction) and measured the extension ratio for the small central region of the sample as well as that for the whole gel. The former is $\alpha_{x}$ in the local level and the latter in the global level. The extension ratio in experiments was controlled globally and the elongation was stopped at global $\alpha_{x}$ of 1.1 in all experiments. The corresponding value of local $\alpha_{x}$ ranged from 1.12 to 1.15 , showing that the local $\alpha_{x}$ is a little larger than the global one. The width determined by experiments is considered to be affected by the transverse mode of the diffusion. This means the boundaries of the gels are actually curvilinear. However, since the change of $a(t)$ with $t$ was very small and then the boundary can be approximated by the plane, we assumed that the $t$ dependent change of $a(t)$ originated only from the volume change.

As shown in Table I, $E_{0}$ of the gels, which were obtained by stress-strain curves, are almost identical to each other for all PAAm gels, although the value of AG1 is a little smaller compared with those for the others. The ratio of the width in the reference state $\left(a_{\mathrm{r}}\right)$ to the final one $\left(a_{\infty}\right)$, is also shown in Table I. The ratio is almost constant for all the PAAm gels. The longest relaxation time $\left(\tau_{1}\right)$ for PAAm gels in Table I was obtained by the two different ways; one is obtained from the plots of $\log \left(-\mu_{\infty} \varepsilon_{x}-\varepsilon_{y}(t)\right)$ vs. $t$ in the long $t$ region, and the other from the plots of $\log \left(\tilde{\sigma}_{x}(t)-\tilde{\sigma}_{x \infty}\right) v s$. $t$ in the same $t$ range. The values of $\tau_{1}$ obtained by the different ways are almost identical to each other for all the gels. This implies that the stress relaxation is induced by the swelling of the gels. The two values for AG1 are larger than those for the other samples. This may be due to the low value of $E_{0}$ for AG1. The diffusion constant $\left(D_{\mathrm{L}}\right)$ was calculated by $D_{\mathrm{L}}=a_{\mathrm{r}}^{2} /\left(2 \pi^{2} \tau_{1}\right)$ using the values of $a_{\mathrm{r}}$ and $\tau_{1}$ obtained by the stress relaxation experiments. The value of $D_{\mathbf{L}}$ ob- 
tained by experiments, which is tabulated in Table I, ranges from $2.5 \times 10^{-7}$ to $7.4 \times 10^{-7}$ $\mathrm{cm}^{2} \mathrm{~s}^{-1}$. Although the value for AG1 is slightly smaller than those for the other samples, their order of magnitude agrees well with the values of $D_{\mathbf{L}}$ for the PAAm gels reported by the isotropic swelling experiments. ${ }^{27,31,32}$

\section{Swelling Behavior of the Uniaxially Stretched Gels}

The value of the strain in $y$-direction $\left(\varepsilon_{y}\right)$ for AG4 is plotted against the reduced time $\left(t / \tau_{1}\right)$ in Figure 1. It is clear that the absolute value of $\varepsilon_{y}$ decreases with increasing $t$; the width increases as $t$ increases. The levelling-off at long times indicates that the gel under a fixed strain reaches the new swelling equilibrium state. The solid curve in Figure 1 shows the theoretical result calculated from eq 34 with $\mu_{0}=$ $1 / 2$ and $\mu_{\infty}=1 / 6$. The value of $\varepsilon_{x}$ in local level was employed for the calculation of $\varepsilon_{y}$, because the strain in $y$ direction obtained by experiment corresponded to that in the central region of the gels. The values of $\mu_{0}$ and $\mu_{\infty}$ for AG4 were respectively 0.41 and 0.14 , and they are not so far from the ideal value of $\mu_{0}=$ $1 / 2$ and $\mu_{\infty}=1 / 6$. Therefore, we limited the theoretical calculation to the ideal case and no curve fitting for the experimental data was made here. Although the $\varepsilon_{y}$ values on the theoretical curve are small compared with the experimental data, the $t$ dependence of the strain is well described by the theory. The difference of the absolute value between the

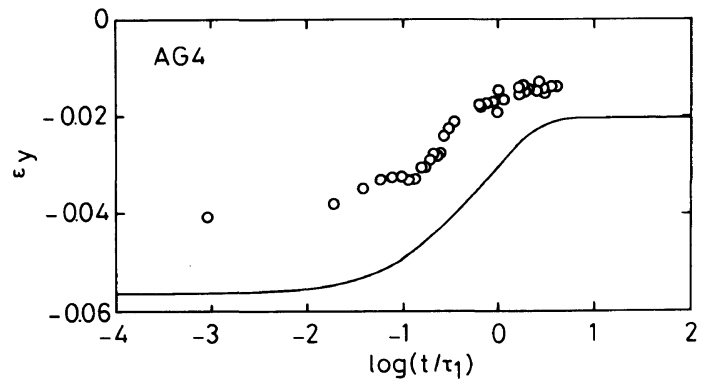

Figure 1. The time dependence of strain perpendicular to the stretched direction $\left(\varepsilon_{y}\right)$ for AG4. experimental data and calculated curve originates from the fact that the absolute value of $\varepsilon_{y}$ obtained by calculation at short times is rather sensitive to $\mu_{0}$, and that in the long time region also sensitive to $\mu_{\infty}$.

Figure 2 shows the double logarithmic plots of $\Delta \varepsilon_{y} / \Delta \varepsilon_{y 0}$ vs. $t / \tau_{1}$ for AG samples. Here,

$$
\begin{gathered}
\Delta \varepsilon_{y}=-\mu_{\infty} \varepsilon_{x}-\varepsilon_{y}(t) \\
\Delta \varepsilon_{y 0}=\left(\mu_{0}-\mu_{\infty}\right) \varepsilon_{x}
\end{gathered}
$$

The values of local $\varepsilon_{x}$ were used for the theoretical calculation of $\varepsilon_{y}$ in eq 34 . The curve in the figure is independent of values of $\mu_{0}$ and $\mu_{\infty}$. Only the $t$ dependence of the strain function appears in the figure. The experimental data points for each gel sample are scattered, but the $t$ dependence seems to be rather well

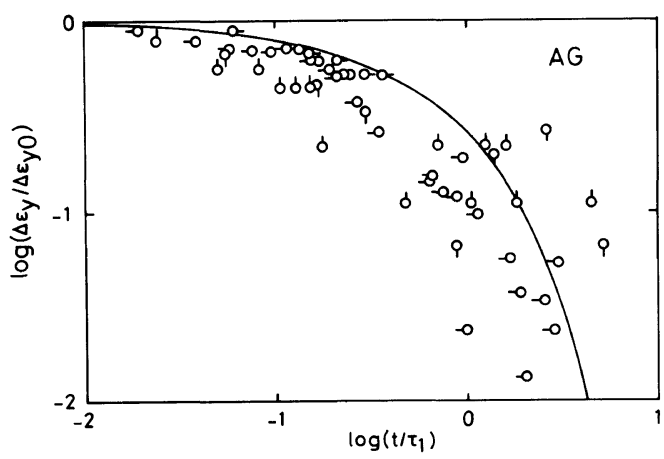

Figure 2. The time dependence of the reduced strain difference in the direction perpendicular to the stretched direction $\left(\Delta \varepsilon_{y} / \Delta \varepsilon_{y 0}\right)$. AG1 (○), AG2 (○), AG3 (९), and AG4 $(-\mathrm{O})$.

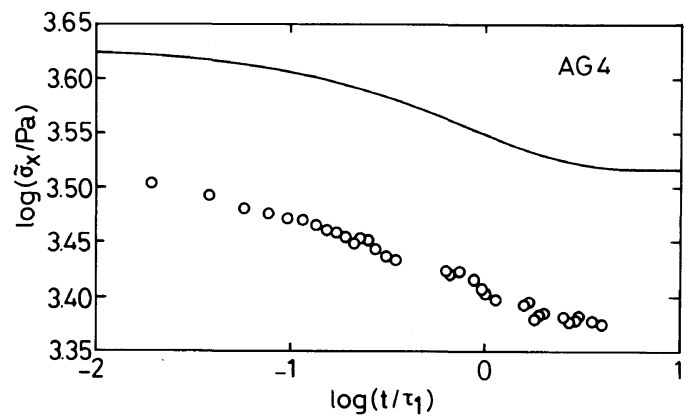

Figure 3. The time dependence of stress in stretched direction $\left(\tilde{\sigma}_{x}\right)$ for AG4. 
described by the theory.

\section{Stress Relaxation of Gels}

Figure 3 shows the double logarithmic plots of $\tilde{\sigma}_{x}(t) v s . t / \tau_{1}$ for AG4. The curve in the figure was calculated from eq 36 by regarding $E_{0}=3.5 \times 10^{4} \mathrm{~Pa}$ as $3 G_{0}$, which means that $\mu_{0}=1 / 2$ is assumed. In addition, $\mu_{\infty}=1 / 6$ is also assumed in the calculation. Since the stress relaxation behavior obtained by experiment is basically determined by the whole gel nature, we used the macroscopic value of $\varepsilon_{x}$ for the calculation, ignoring the non-uniform elongation. The theoretical curve seems not to coincide with the experimental data concerning the stress values, but the curve and the plots are very similar to each other concerning the $t$ dependence of $\tilde{\sigma}_{x}$. Although the difference of $\tilde{\sigma}_{x}$ between theory and experiment mainly originates from the difference of $\mu_{0}$ and $\mu_{\infty}$, as in the case of the absolute value of $\varepsilon_{y}$, the difference at short times, if we see it in the value of $E_{0}$, is about $25 \%$, and that in the long time region is smaller. The value of $\tilde{\sigma}_{x}$ in the long time region agrees rather well with the expected value in equilibrium (the levelled-off value at long times in the figure).

Figure 4 shows the double logarithmic plots of $\Delta \tilde{\sigma}_{x} / \Delta \tilde{\sigma}_{x 0}$ vs. $t / \tau_{1}$ for AG samples. $\Delta \tilde{\sigma}_{x}$ and $\Delta \tilde{\sigma}_{x 0}$ are

$$
\Delta \tilde{\sigma}_{x}=\tilde{\sigma}_{x}(t)-\tilde{\sigma}_{x \infty}
$$

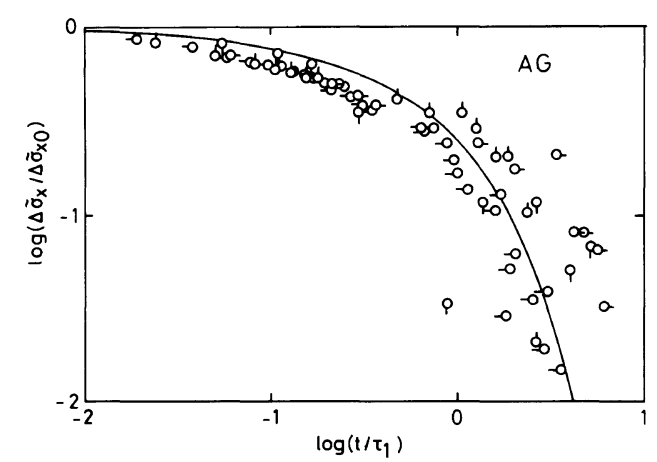

Figure 4. The time dependence of the reduced stress difference in the stretched direction $\left(\Delta \tilde{\sigma}_{x} / \Delta \tilde{\sigma}_{x 0}\right)$. AG1 (○), AG2 (○), AG3 (○), and AG4 (-○).

$$
\Delta \tilde{\sigma}_{x 0}=\tilde{\sigma}_{x 0}-\tilde{\sigma}_{x \infty}
$$

Here, $\tilde{\sigma}_{x 0}$ and $\tilde{\sigma}_{x \infty}$ are the initial and final stresses. Only the $t$ dependence of the stress function is observed in the reduced plots shown here. The curve in the figure, which was calculated using the expression of $\tilde{\sigma}_{x}(t)$ in eq 36 , represents the results of the theoretical calculation. The macroscopic values of $\varepsilon_{x}$ were employed in the calculation. Although the data points are rather scattered, especially at long times, they are located around the curve, suggesting that the $t$ dependence of the stress function is also well described with the theory.

\section{CONCLUSIONS}

The Poisson's ratio $(\mu)$ and the stress value $\tilde{\sigma}_{x \infty}$ in the equilibrium after uniaxial elongation have been shown. The value of $\mu$ at long $t\left(\mu_{\infty}\right)$, which determines the degree of swelling under tension was approximated to be $1 / 6$ for small applied strains, and $\tilde{\sigma}_{x \infty}=(7 / 3) G_{0} \varepsilon_{x}$ was derived. The swelling kinetics for the rectangular gels, after uniaxial elongation, was shown. The stress relaxation from the initial to final values, obeyed the same kinetics as the swelling. The experimental results for PAAm gels, both in equilibrium properties and kinetics, agreed well with the theory.

\section{REFERENCES}

1. J. D. Andrade, "Hydrogels for Medical and Related Applications," American Chemical Society, Washington D.C., 1976.

2. K. Tamura, O. Ike, S. Hitomi, J. Isobe, Y. Shimizu, and M. Nambu, Trans. Am. Soc. Artif. Inter. Organs, 32, 605 (1986).

3. S.-H. Hyon, W. I. Cha, and Y. Ikada, Polym. Bull., 22, 119 (1989).

4. Y. Hirokawa and T. Tanaka, J. Chem. Phys., 81, 6379 (1984).

5. S. Hirotsu, J. Phys. Soc. Jpn., 56, 233 (1987).

6. S. Hirotsu, Y. Hirokawa, and T. Tanaka, J. Chem. Phys., 87, 1392 (1987).

7. S. Hirotsu and A. Onuki, J. Phys. Soc. Jpn., 58, 1508 (1989).

8. H. Inomata, S. Goto, and S. Saito, Macromolecules, 
23, 4887 (1990)

9. K. Otake, H. Inomata, M. Konno, and S. Saito, Macromolecules, 23, 283 (1990).

10. P. J. Flory, "Principles of Polymer Chemistry," Cornell University Press, Ithaca, 1953.

11. R. W. Brotzman and B. E. Eichinger, Macromolecules, 14, 1445 (1981).

12. R. W. Brotzman and B. E. Eichinger, Macromolecules, 16, 1131 (1983).

13. N. A. Neuburger and B. E. Eichinger, Macromolecules, 21, 3060 (1988).

14. F. Horkay, Macromolecules, 22, 2007 (1989).

15. F. Horkay, A.-M. Hecht, and E. Geissler, J. Chem. Phys., 912706 (1989).

16. A.-M. Hecht, F. Horkay, E. Geissler, and M. Zrinyi, Polym. Cummun., 31, 53 (1990).

17. Y. Rabin and E. T. Samulski, Macromolecules, 25, 2985 (1992).

18. S. Daoudi, J. Phys. (Paris), 38, 1301 (1977).

19. E. Geissler and A.-M. Hecht, Macromolecules, 13, 1276 (1980).

20. K. Nishinari, M. Watase, K. Ogino, and M. Nambu, Polym. Commun., 24, 345 (1983).

21. E. Geissler and A.-M. Hecht, Macromolecules, 14,
185 (1981)

22. E. Geissler, A.-M. Hecht, F. Horkay, and M. Zrinyi, Macromolecules, 21, 2594 (1988).

23. M. Watase and K. Nishinari, Polym. J., 21, 567 (1989).

24. S.-H. Hyon, W. I. Cha, and Y. Ikada, Kobunshironbunshu, 46, 673 (1989).

25. T. Takigawa, H. Kashihara, and T. Masuda, Polym. Bull., 24, 613 (1990).

26. S. Alexander and Y. Rabin, J. Phys.: Condens. Matter, 2, SA313 (1990).

27. T. Tanaka, L. Hocker, and G. Benedek, J. Chem. Phys., 59, 5151 (1972).

28. T. Tanaka and D. J. Fillmore, J. Chem. Phys., 70, 1214 (1979).

29. K. Urayama, T. Takigawa, and T. Masuda, Macromolecules, 26, 3092 (1993).

30. L. D. Landau and E. M. Lifshitz, "Theory of Elasticity," Nauka, Moscow, 1987.

31. A. Peters and S. J. Candau, Macromolecules, 19, 1952 (1986).

32. A. Peters and S. J. Candau, Macromolecules, 21, 2278 (1988). 